Ann. Scient. Éc. Norm. Sup.

$4^{\text {e }}$ série, t. 45,2012, p. 511 à 534

\title{
ALGEBRAIC HOMOTOPY CLASSES OF RATIONAL FUNCTIONS
}

\author{
B Y Christophe CAZANAVE
}

Abstract. - Let $k$ be a field. We compute the set $\left[\mathbf{P}^{1}, \mathbf{P}^{1}\right]^{\mathrm{N}}$ of naive homotopy classes of pointed $k$-scheme endomorphisms of the projective line $\mathbf{P}^{1}$. Our result compares well with Morel's computation in [11] of the group $\left[\mathbf{P}^{1}, \mathbf{P}^{1}\right]^{\mathbf{A}^{1}}$ of $\mathbf{A}^{1}$-homotopy classes of pointed endomorphisms of $\mathbf{P}^{1}$ : the set $\left[\mathbf{P}^{1}, \mathbf{P}^{1}\right]^{\mathrm{N}}$ admits an a priori monoid structure such that the canonical map $\left[\mathbf{P}^{1}, \mathbf{P}^{1}\right]^{\mathrm{N}} \rightarrow\left[\mathbf{P}^{1}, \mathbf{P}^{1}\right]^{\mathbf{A}^{1}}$ is a group completion.

RÉSUmÉ. - Soit $k$ un corps. Nous déterminons l'ensemble $\left[\mathbf{P}^{1}, \mathbf{P}^{1}\right]^{\mathrm{N}}$ des classes d'homotopie nä̈ve d'endomorphismes pointés de $k$-schémas de la droite projective $\mathbf{P}^{1}$. Notre résultat se compare bien avec le calcul de Morel [11] du groupe $\left[\mathbf{P}^{1}, \mathbf{P}^{1}\right]^{\mathbf{A}^{1}}$ des classes d' $\mathbf{A}^{1}$-homotopie d'endomorphismes pointés de $\mathbf{P}^{1}$ : l'ensemble $\left[\mathbf{P}^{1}, \mathbf{P}^{1}\right]^{\mathrm{N}}$ admet a priori une structure de monoïde pour laquelle l'application canonique $\left[\mathbf{P}^{1}, \mathbf{P}^{1}\right]^{\mathrm{N}} \rightarrow\left[\mathbf{P}^{1}, \mathbf{P}^{1}\right]^{\mathbf{A}^{1}}$ est une complétion en groupe.

\section{Introduction}

The work of Fabien Morel and Vladimir Voevodsky on $\mathbf{A}^{1}$-homotopy theory $[9,12]$ provides a convenient framework to do algebraic topology in the setting of algebraic geometry. More precisely, for a fixed field $k$, Morel and Voevodsky defined an appropriate category of spaces, say $\delta p$, containing the category of smooth algebraic $k$-varieties as a full subcategory, which they endowed with a suitable model structure, in the sense of Quillen's homotopical algebra [13]. Thus, given two spaces $X$ and $Y$ in $\delta p$ (resp. two pointed spaces), the set $\{X, Y\}^{\mathbf{A}^{1}}$ (resp. the set $[X, Y]^{\mathbf{A}^{1}}$ ) of $\mathbf{A}^{1}$-homotopy classes of unpointed morphisms (resp. of pointed morphisms) from $X$ to $Y$ is well defined and has all the properties an algebraic topologist can expect. However, for concrete $X$ and $Y$, these sets are in general hard to compute.

At the starting point of $\mathbf{A}^{1}$-homotopy theory is the notion of naive homotopy ${ }^{(1)}$ between two morphisms in $\$ p$. First introduced by Karoubi and Villamayor [6], this notion mimics

This research was partially supported by the project ANR blanc BLAN08-2_338236, HGRT.

(1) In [12], the authors use the terminology "elementary homotopy". 
the usual one of homotopy between topological maps, replacing the unit interval $[0,1]$ by its algebraic analogue, the affine line $\mathbf{A}^{1}$.

Definition 1.1. - Let $X$ and $Y$ be two spaces in $\delta p$. A naive homotopy is a morphism in $\delta p$

$$
F: X \times \mathbf{A}^{1} \longrightarrow Y \text {. }
$$

The restriction $\sigma(F):=F_{\mid X \times\{0\}}$ is the source of the homotopy and $\tau(F):=F_{\mid X \times\{1\}}$ is its target. When $X$ and $Y$ have base points, say $x_{0}$ and $y_{0}$, we say that $F$ is pointed if its restriction to $\left\{x_{0}\right\} \times \mathbf{A}^{1}$ is constant equal to $y_{0}$.

With this notion, one defines the set $\{X, Y\}^{\mathrm{N}}$ (resp. the set $[X, Y]^{\mathrm{N}}$ ) of unpointed (resp. of pointed) naive homotopy classes of morphisms from $X$ to $Y$ as the quotient of the set of unpointed (resp. of pointed) morphisms by the equivalence relation generated by unpointed (resp. by pointed) naive homotopies. These sets are sometimes easier to compute than their $\mathbf{A}^{1}$ analogues, but they are not very well behaved. There is a canonical comparison map

$$
[X, Y]^{\mathrm{N}} \longrightarrow[X, Y]^{\mathbf{A}^{1}}
$$

which in general is far from being a bijection. In this article, we study a particular situation where this map has a noteworthy behavior.

Let $k$ be a base field. We focus on the set of pointed homotopy classes of $k$-scheme endomorphisms of the projective line $\mathbf{P}^{1}$ with base point $\infty:=[1: 0]$. The set $\left[\mathbf{P}^{1}, \mathbf{P}^{1}\right]^{\mathbf{A}^{1}}$ is computed by Fabien Morel in [11]. Note that, as the source space $\mathbf{P}^{1}$ is homotopy equivalent in $S p$ to a suspension (see Lemma 3.20), the set $\left[\mathbf{P}^{1}, \mathbf{P}^{1}\right]^{\mathbf{A}^{1}}$ is endowed with a natural group structure, whose law is denoted by $\oplus^{\mathbf{A}^{1}}$.

On the other hand, by interpreting endomorphisms of $\mathbf{P}^{1}$ as rational functions, we define a monoid law $\oplus^{\mathrm{N}}$ on $\left[\mathbf{P}^{1}, \mathbf{P}^{1}\right]^{\mathrm{N}}$. Using this additional structure and a classical construction due to Bézout, we can give an explicit description of $\left[\mathbf{P}^{1}, \mathbf{P}^{1}\right]^{\mathrm{N}}$. Morel's computation combined with ours then leads to the following striking result.

THeOrem 1.2. - The canonical map

$$
\left(\left[\mathbf{P}^{1}, \mathbf{P}^{1}\right]^{\mathrm{N}}, \oplus^{\mathrm{N}}\right) \longrightarrow\left(\left[\mathbf{P}^{1}, \mathbf{P}^{1}\right]^{\mathbf{A}^{1}}, \oplus^{\mathbf{A}^{1}}\right)
$$

is a group completion.

\section{Overview of the paper}

Section 2 reviews the classical correspondence between scheme endomorphisms of the projective line and rational functions. This leads to a description of $\left[\mathbf{P}^{1}, \mathbf{P}^{1}\right]^{\mathrm{N}}$ as a set of algebraic homotopy classes of rational functions with coefficients in the field $k$.

Section 3 is the core of the article. In $\S 3.1$, we define a monoid structure on the scheme $\mathcal{F}$ of pointed rational functions. Through the correspondence of Section 2, it induces the monoid law $\oplus^{\mathrm{N}}$ which appears in Theorem 1.2. Then $\S 3.2$ reviews a classical construction due to Bézout, which associates to any rational function a non-degenerate symmetric $k$-bilinear form. We use it to define a homotopy invariant of rational functions taking values in some set of equivalence classes of symmetric $k$-bilinear forms. Our main result, stated in $\S 3.3$, shows that this invariant distinguishes exactly all the homotopy classes of rational functions. The 
proof is given in $\S 3.4$. Finally, $\$ 3.5$ compares our result to the actual $\mathbf{A}^{1}$-homotopy classes of Morel, as in Theorem 1.2.

Section 4 discusses natural extensions of the previous computation. We first give a similar description of the set of unpointed naive homotopy classes of endomorphisms of $\mathbf{P}^{1}$ in $\$ 4.1$. Next, in $\S 4.2$, we study the composition of endomorphisms of $\mathbf{P}^{1}$ in terms of our description of $\left[\mathbf{P}^{1}, \mathbf{P}^{1}\right]^{\mathrm{N}}$. Finally, in $\S 4.3$, we compute the set $\left[\mathbf{P}^{1}, \mathbf{P}^{d}\right]^{\mathrm{N}}$ of pointed naive homotopy classes of morphisms from $\mathbf{P}^{1}$ to $\mathbf{P}^{d}$ for every integer $d \geqslant 2$. Not surprisingly, this case is easier than the case $d=1$. The result still compares well with Morel's computation of the actual $\mathbf{A}^{1}$-homotopy classes.

The article ends on an appendix proving the compatibility of the law $\oplus^{\mathrm{N}}$ on $\left[\mathbf{P}^{1}, \mathbf{P}^{1}\right]^{\mathrm{N}}$ with that $\oplus^{\mathbf{A}^{1}}$ on $\left[\mathbf{P}^{1}, \mathbf{P}^{1}\right]^{\mathbf{A}^{1}}$. This is a crucial part of the comparison of our results to those of Morel.

\section{Acknowledgements}

The material presented here constitutes the first part of my $\mathrm{PhD}$ thesis [3]. The main result was first announced in the note [2] when $\operatorname{char}(k) \neq 2$. I am very much indebted to Jean Lannes for his precious and generous help. I am also grateful to the topology group of Bonn Universität for its hospitality while this article was written.

\section{Rational functions and naive homotopies}

We review the classical correspondence between pointed $k$-scheme endomorphisms of the projective line $\left(\mathbf{P}^{1}, \infty\right)$ and pointed rational functions with coefficients in $k$. Similarly, naive homotopies have a description in terms of pointed rational functions with coefficients in the $\operatorname{ring} k[T]$.

Definition 2.1. - For an integer $n \geqslant 1$, the scheme $\mathcal{F}_{n}$ of pointed degree $n$ rational functions is the open subscheme of the affine space $\mathbf{A}^{2 n}=\operatorname{Spec} k\left[a_{0}, \ldots, a_{n-1}, b_{0}, \ldots, b_{n-1}\right]$ complementary to the hypersurface of equation ${ }^{(2)}$

$$
\operatorname{res}_{n, n}\left(X^{n}+a_{n-1} X^{n-1}+\cdots+a_{0}, b_{n-1} X^{n-1}+\cdots+b_{0}\right)=0 .
$$

By convention, $\mathcal{F}_{0}:=\operatorname{Spec} k$.

REMARK 2.2. - Let $R$ be a $k$-algebra and $n$ a non-negative integer. By the very definition, an $R$-point of $\mathcal{F}_{n}$ is a pair of polynomials $(A, B) \in R[X]^{2}$, where

- $A$ is monic of degree $n$,

- $B$ is of degree strictly less than $n$,

- the scalar $\operatorname{res}_{n, n}(A, B)$ is invertible in $R$.

Such an element is denoted by $\frac{A}{B}$ and is called a pointed degree $n$ rational function with coefficients in $R$. In the sequel, it is useful to remark that given $A$ and $B$ as above, the

(2) The notation $\operatorname{res}_{n, n}(A, B)$ stands for the resultant of the polynomials $A$ and $B$ with conventions as in $\left[1, \S 6, n^{\circ} 6, I V\right]$. 
condition $\operatorname{res}_{n, n}(A, B) \in R^{\times}$is equivalent to the existence of a (necessarily unique) Bézout relation

$$
A U+B V=1
$$

with $U$ and $V$ polynomials in $R[X]$ such that $\operatorname{deg} V \leqslant n-1$ (and $\operatorname{deg} U \leqslant n-2$ if $n \neq 0$ ).

Pointed $k$-scheme morphisms $\mathbf{P}^{1} \rightarrow \mathbf{P}^{1}$ and pointed naive homotopies $F: \mathbf{P}^{1} \times \mathbf{A}^{1}=$ $\mathbf{P}_{k[T]}^{1} \rightarrow \mathbf{P}^{1}$ are then described in terms of rational functions as follows.

Proposition 2.3. - Let $R=k$ or $R=k[T]$. The datum of a pointed $k$-scheme morphism $f: \mathbf{P}_{R}^{1} \longrightarrow \mathbf{P}^{1}$ is equivalent to the datum of a non-negative integer $n$ and of an element $\frac{A}{B} \in \mathcal{F}_{n}(R)$. The integer $n$ is called the degree of $f$ and is denoted $\operatorname{deg}(f)$; the scalar $\operatorname{res}_{n, n}(A, B) \in R^{\times}=k^{\times}$is called the resultant of $f$ and is denoted $\operatorname{res}(f)$.

Proof. - This follows from the usual description of morphisms to a projective space (using the fact that the ring $R$ is a UFD).

ExAmPle 2.4. - Let $n$ be a positive integer and $b_{0}$ be a unit in $k^{\times}$.

1. A polynomial $\frac{X^{n}+a_{n-1} X^{n-1}+\cdots+a_{0}}{b_{0}}$ is homotopic to its leading term $\frac{X^{n}}{b_{0}}$.

2. Let $B$ be a polynomial of degree $\leqslant n-1$ such that $B(0)=b_{0}$. Then $\frac{X^{n}}{B} \stackrel{p}{\sim} \frac{X^{n}}{b_{0}}$.

In general, given a random rational function, it is not a priori easy to find non-trivial homotopies. In Remark 3.2(2), we will indicate a way of producing some such homotopies.

Definition 2.5. - Let $f$ and $g$ be two pointed rational functions over $k$. We say that $f$ and $g$ are in the same pointed naive homotopy class, and we write $f \stackrel{\text { p }}{\sim} g$, if there exists a finite sequence of pointed homotopies, say $\left(F_{i}\right)$ with $0 \leqslant i \leqslant N$, such that

$-\sigma\left(F_{0}\right)=f$ and $\tau\left(F_{N}\right)=g$;

- for every $0 \leqslant i \leqslant N-1$, we have $\tau\left(F_{i}\right)=\sigma\left(F_{i+1}\right)$.

The set of pointed naive homotopy classes $\left[\mathbf{P}^{1}, \mathbf{P}^{1}\right]^{\mathrm{N}}$ is thus the quotient set $\coprod_{n \geqslant 0} \mathcal{F}_{n}(k) / \mathrm{p}$.

Note that Proposition 2.3 implies that two pointed rational functions which are in the same pointed naive homotopy class have same degree (and also same resultant). In particular, the set $\left[\mathbf{P}^{1}, \mathbf{P}^{1}\right]^{\mathrm{N}}$ splits into the disjoint union of its degreewise components

$$
\left[\mathbf{P}^{1}, \mathbf{P}^{1}\right]^{\mathrm{N}}=\coprod_{n \geqslant 0}\left[\mathbf{P}^{1}, \mathbf{P}^{1}\right]_{n}^{\mathrm{N}}
$$

REMARK 2.6. - It is convenient to reformulate the preceding discussion in terms of the "naive connected components" of the scheme of pointed rational functions.

For $\mathscr{G}: \operatorname{Alg}_{k} \longrightarrow$ Set a functor from the category of $k$-algebras to that of sets, recall that $\pi_{0}^{\mathrm{N}} \mathscr{G}: \mathrm{Alg}_{k} \longrightarrow$ Set is the functor which assigns to a $k$-algebra $R$ the coequalizer of the double-arrow $\mathscr{G}(R[T]) \rightrightarrows \mathscr{G}(R)$ given by evaluation at $T=0$ and $T=1$. For every non-negative integer $n$, Proposition 2.3 gives a bijection

$$
\left[\mathbf{P}^{1}, \mathbf{P}^{1}\right]_{n}^{\mathrm{N}} \simeq\left(\pi_{0}^{\mathrm{N}} \mathcal{F}_{n}\right)(k) .
$$

Note that by functoriality a $k$-scheme morphism $\phi: \mathcal{F}_{n} \longrightarrow \chi$ induces a homotopy invariant $\pi_{0}^{\mathrm{N}}(\phi)(k):\left(\pi_{0}^{\mathrm{N}} \mathcal{F}_{n}\right)(k) \longrightarrow\left(\pi_{0}^{\mathrm{N}} \chi\right)(k)$. 


\section{Homotopy classes of rational functions}

\subsection{Additions of rational functions}

An important feature in our results is the existence a priori of a monoid structure on the set of naive homotopy classes $\left[\mathbf{P}^{1}, \mathbf{P}^{1}\right]^{\mathrm{N}}$. It comes as a byproduct of a graded monoid structure on the disjoint union scheme $\mathcal{F}:=\coprod_{n \geqslant 0} \mathcal{F}_{n}$.

Such a structure on $\mathcal{F}$ consists of a family of morphisms $\mathcal{F}_{n_{1}} \times \mathcal{F}_{n_{2}} \longrightarrow \mathcal{F}_{n_{1}+n_{2}}$, indexed by pairs $\left(n_{1}, n_{2}\right)$ of non-negative integers and subject to an associativity condition. Given a pair of non-negative integers $\left(n_{1}, n_{2}\right)$, the above structural morphism is more easily described on the level of functors of points, that is as a natural transformation of functors from $\mathrm{Alg}_{k}$ to Set:

$$
\mathcal{F}_{n_{1}}(-) \times \mathcal{F}_{n_{2}}(-) \longrightarrow \mathcal{F}_{n_{1}+n_{2}}(-)
$$

Let $R$ be a $k$-algebra. Two rational functions $\frac{A_{i}}{B_{i}} \in \mathcal{F}_{n_{i}}(R)$, for $i=1,2$, uniquely define two pairs $\left(U_{i}, V_{i}\right)$ of polynomials of $R[X]$ with $\operatorname{deg} U_{i} \leqslant n_{i}-2$ and $\operatorname{deg} V_{i} \leqslant n_{i}-1$ and satisfying Bézout identities $A_{i} U_{i}+B_{i} V_{i}=1$ (see Remark 2.2). Define polynomials $A_{3}, B_{3}, U_{3}$ and $V_{3}$ by setting ${ }^{(3)}$ :

$$
\left[\begin{array}{cc}
A_{3} & -V_{3} \\
B_{3} & U_{3}
\end{array}\right]:=\left[\begin{array}{cc}
A_{1} & -V_{1} \\
B_{1} & U_{1}
\end{array}\right] \cdot\left[\begin{array}{cc}
A_{2} & -V_{2} \\
B_{2} & U_{2}
\end{array}\right]
$$

As $\left[\begin{array}{cc}A_{1} & -V_{1} \\ B_{1} & U_{1}\end{array}\right]$ and $\left[\begin{array}{cc}A_{2} & -V_{2} \\ B_{2} & U_{2}\end{array}\right]$ belong to $\mathbf{S L}_{2}(k[T])$, this is also the case for $\left[\begin{array}{cc}A_{3}-V_{3} \\ B_{3} & U_{3}\end{array}\right]$, which gives a Bézout relation for $A_{3}$ and $B_{3}$. Moreover, observe that $A_{3}$ is monic of degree $n_{1}+n_{2}$ and that $\operatorname{deg}\left(B_{3}\right)<n_{1}+n_{2}$. So $\frac{A_{3}}{B_{3}}$ is an $R$-point of $\mathcal{F}_{n_{1}+n_{2}}$. We write

$$
\frac{A_{1}}{B_{1}} \oplus^{\mathrm{N}} \frac{A_{2}}{B_{2}}:=\frac{A_{3}}{B_{3}} .
$$

The associativity of matrix multiplication implies that of the law $\oplus^{\mathrm{N}}$.

Proposition 3.1. - The above morphisms define a graded monoid structure on $\mathcal{F}:=\coprod_{n \geqslant 0} \mathcal{F}_{n}$.

REMARK 3.2. - 1. The above monoid structure on $\mathcal{F}$ induces a graded monoid structure on its connected components $\left(\pi_{0}^{\mathrm{N}} \mathcal{F}\right)(k):=\coprod_{n \geqslant 0}\left(\pi_{0}^{\mathrm{N}} \mathcal{F}_{n}\right)(k)$, and thus on $\left[\mathbf{P}^{1}, \mathbf{P}^{1}\right]^{\mathrm{N}}$. The monoid law on these sets is again denoted by $\oplus^{\mathrm{N}}$.

2. The $\oplus^{\mathrm{N}}$-sum of "trivial" homotopies is usually a "non trivial" homotopy.

3. Although we use an additive notation $\oplus^{\mathrm{N}}$ for the monoid law on $\mathcal{F}$, we stress that it non commutative. However, Corollary 3.7 shows that the monoid $\left(\left[\mathbf{P}^{1}, \mathbf{P}^{1}\right]^{\mathrm{N}}, \oplus^{\mathrm{N}}\right)$ is abelian.

(3) The dot in the right-hand term stands for the usual matrix multiplication. 
Example 3.3. - Let $R$ be a $k$-algebra, $P \in R[X]$ be a monic polynomial, $b_{0} \in R^{\times}$be a unit and $\frac{A}{B} \in \mathcal{F}(R)$ be a pointed rational function. Then one has:

$$
\frac{P}{b_{0}} \oplus^{\mathrm{N}} \frac{A}{B}=\frac{A P-\frac{B}{b_{0}}}{b_{0} A}=\frac{P}{b_{0}}-\frac{1}{b_{0}^{2} \frac{A}{B}} .
$$

In particular, for rational functions with coefficients in the field $k$, the law $\oplus^{\mathrm{N}}$ is easily described in terms of "twisted" continued fraction expansions as follows.

A pointed rational fraction $f \in \mathcal{F}_{n}(k)$ admits a unique "twisted" expansion of the following form:

$$
f=\frac{P_{0}}{b_{0}}-\frac{1}{b_{0}^{2}\left(\frac{P_{1}}{b_{1}}-\frac{1}{b_{1}^{2}(\ldots)}\right)},
$$

for monic polynomials $P_{i} \in k[X]$ of positive degrees and scalars $b_{i} \in k^{\times}$. (Such expansions always end, as the sum of the degrees of the $P_{i}$ equals the degree of $f$.) The twisted continued fraction expansion of a sum $f \oplus^{\mathrm{N}} g$ is then the concatenation of the expansions of $f$ and $g$.

Note that every $f \in \mathcal{F}_{n}(k)$ is tautologically the $\oplus^{\mathrm{N}}$-sum of the polynomials appearing in its twisted continued fraction expansion:

$$
f=\frac{P_{0}}{b_{0}} \oplus^{\mathrm{N}} \frac{P_{1}}{b_{1}} \oplus^{\mathrm{N}} \cdots \oplus^{\mathrm{N}} \frac{P_{r}}{b_{r}} .
$$

\subsection{The Bézout form}

In the 18th century, Bézout described a way to associate to every rational function a nondegenerate symmetric matrix ${ }^{(4)}$. In modern terminology, Bézout described, for every positive integer $n$, a scheme morphism

$$
\text { Béz }{ }_{n}: \mathcal{F}_{n} \longrightarrow \phi_{n}
$$

where $\phi_{n}$ is the scheme of non-degenerate $(n \times n)$ symmetric matrices. The associated homotopy invariants $\left(\pi_{0}^{\mathrm{N}} \mathcal{F}_{n}\right)(k) \stackrel{\pi_{0}^{\mathrm{N}} \mathrm{Béz}_{n}}{\longrightarrow}\left(\pi_{0}^{\mathrm{N}} \phi_{n}\right)(k)$ are central in our study.

Definition 3.4. - Let $R$ be a ring, $n$ be a positive integer and $f=\frac{A}{B}$ be an element of $\mathcal{F}_{n}(R)$.

The polynomial $A(X) B(Y)-A(Y) B(X) \in R[X, Y]$ is divisible by $X-Y$. Let

$$
\delta_{A, B}(X, Y):=\frac{A(X) B(Y)-A(Y) B(X)}{X-Y}=: \sum_{1 \leqslant p, q \leqslant n} c_{p, q} X^{p-1} Y^{q-1} .
$$

Observe that the coefficients of $\delta_{A, B}(X, Y)$ are symmetric in the sense that one has

$$
c_{p, q}=c_{q, p} \quad \forall 1 \leqslant p, q \leqslant n .
$$

The Bézout form of $f$ is the symmetric bilinear form over $R^{n}$ whose Gram matrix is the $(n \times n)$-symmetric matrix $\left[c_{p, q}\right]_{1 \leqslant p, q \leqslant n}$. We denote it Béz $z_{n}(A, B)$ or Béz $z_{n}(f)$ and Bézout's formula:

$$
\operatorname{det} \operatorname{Bé}_{n}(f)=(-1)^{\frac{n(n-1)}{2}} \operatorname{res}(f)
$$

shows that this form is non-degenerate.

(4) This construction is sometimes called the "Bezoutian", but we prefer the terminology "Bézout form". 
The above construction describes for every positive integer a natural transformation of functors $\mathcal{F}_{n}(-) \longrightarrow \phi_{n}(-)$ and thus a morphism of schemes

$$
\text { Béz }{ }_{n}: \mathcal{F}_{n} \longrightarrow \phi_{n} \text {. }
$$

Remark 3.5. - A more conceptual definition of the Bézout form in terms of Serre duality can be found in [5, Example III.4.8]

\subsection{The main theorem}

The Bézout invariants distinguish exactly all the naive homotopy classes of rational functions. More precisely, we have the following.

THEOREM 3.6. - The following map is an isomorphism of graded monoids:

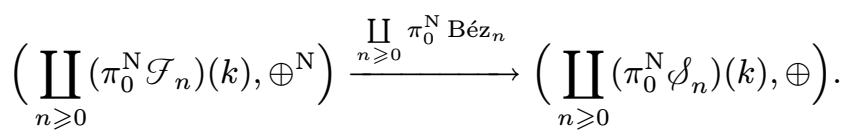

The proof of Theorem 3.6 is postponed until 33.4 .

COROLlary 3.7. - The monoid $\left(\coprod_{n \geqslant 0}\left(\pi_{0}^{\mathrm{N}} \phi_{n}\right)(k), \oplus\right)$ is abelian, and thus so is $\left(\left[\mathbf{P}^{1}, \mathbf{P}^{1}\right]^{\mathrm{N}}, \oplus^{\mathrm{N}}\right)$.

To make Theorem 3.6 more explicit, we precise the sets of value $\left(\pi_{0}^{\mathrm{N}} \phi_{n}\right)(k)$ of the invariants.

Definition 3.8. - $\quad$ 1. The Witt monoid of the field $k$ is the monoid, for the orthogonal $\operatorname{sum} \oplus$, of isomorphism classes of non-degenerate symmetric $k$-bilinear forms. We denote it $\mathrm{MW}(k)$.

2. Let $\mathrm{MW}^{\mathrm{s}}(k)$ be the monoid of stable isomorphism classes of non-degenerate symmetric $k$-bilinear forms. By definition, this is the quotient of $\mathrm{MW}(k)$ where two forms $b$ and $b^{\prime}$ are to be identified if there exists a form $b^{\prime \prime}$ such that $b \oplus b^{\prime \prime} \simeq b^{\prime} \oplus b^{\prime \prime}$. It comes with a natural grading induced by the rank, and for every positive integer $n$, we denote by $\mathrm{MW}_{n}^{\mathrm{s}}(k)$ the degree $n$ component of $\mathrm{MW}^{\mathrm{s}}(k)$. (Note that when $\operatorname{char}(k) \neq 2$ then $\mathrm{MW}^{\mathrm{s}}(k)=\mathrm{MW}(k)$.)

3. The Grothendieck-Witt group $\mathrm{GW}(k)$ is the Grothendieck group of the monoid $\mathrm{MW}^{\mathrm{s}}(k)$.

Proposition 3.9. - Let $n$ be a positive integer.

1. The canonical quotient map $q_{n}: \phi_{n}(k) \longrightarrow \mathrm{MW}_{n}^{\mathrm{s}}(k)$ factors through $\left(\pi_{0}^{\mathrm{N}} \phi_{n}\right)(k)$ :

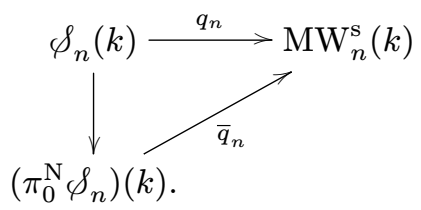


2. Let $\mathrm{MW}_{n}^{\mathrm{s}}(k) \underset{k^{\times}}{\times}{ }_{k^{\times 2}} k^{\times}$denote the canonical fibre product where $\mathrm{MW}_{n}^{\mathrm{s}}(k) \longrightarrow k^{\times} / k^{\times 2}$ is the discriminant map. Then the map

$$
\left(\coprod_{n \geqslant 0}\left(\pi_{0}^{\mathrm{N}} \phi_{n}\right)(k), \oplus\right) \stackrel{\underset{n \geqslant 0}{\amalg} \bar{q}_{n} \times \operatorname{det}}{\longrightarrow}\left(\coprod_{n \geqslant 0} \operatorname{MW}_{n}^{\mathrm{s}}(k) \underset{k^{\times} / k^{\times 2}}{\times} k^{\times}, \oplus\right)
$$

is a monoid isomorphism. (Above, the right-hand term is endowed with the canonical monoid structure induced by the orthogonal sum in $\mathrm{MW}^{\mathrm{s}}(k)$ and the product in $\left.k^{\times}\right)$.

Proof. - This is certainly well-known to specialists (at least when char $(k) \neq 2$ ). We would like to advertise a proof due to Gerstein, based on a very elegant use of Hermite inequality for symmetric $k[T]$-bilinear forms. The reader is referred to the very clear exposition in [7, §VII.3] (see in particular Remark VII.3.14 when $\operatorname{char}(k)=2$ ).

Theorem 3.6 and Proposition 3.9 together give the following explicit description of $\left[\mathbf{P}^{1}, \mathbf{P}^{1}\right]^{\mathrm{N}}$.

COROLlaRY 3.10. - There is a canonical isomorphism of graded monoids:

$$
\left(\left[\mathbf{P}^{1}, \mathbf{P}^{1}\right]^{\mathrm{N}}, \oplus^{\mathrm{N}}\right) \simeq\left(\coprod_{n \geqslant 0} \operatorname{MW}_{n}^{\mathrm{s}}(k) \underset{k^{\times} / k^{\times 2}}{\times} k^{\times}, \oplus\right) .
$$

EXAmPLE 3.11. - 1. When $k$ is algebraically closed, we have an isomorphism of monoids

$$
\left[\mathbf{P}^{1}, \mathbf{P}^{1}\right]^{\mathrm{N}} \underset{\operatorname{deg} \times \operatorname{res}}{\simeq} \mathbf{N} \times k^{\times} .
$$

The Bézout invariants reduce to the resultant and degree invariants.

2. When $k$ is the field of real numbers $\mathbf{R}$, we have an isomorphism of monoids:

$$
\left[\mathbf{P}^{1}, \mathbf{P}^{1}\right]^{\mathrm{N}} \underset{(\text { signoBéz }) \times \text { res }}{\simeq}(\mathbf{N} \times \mathbf{N}) \times \mathbf{R}^{\times},
$$

sign denoting the signature of a real symmetric bilinear form. In this case, the Bézout invariant is sharper than the resultant and the degree invariants.

\subsection{Proof of Theorem 3.6}

Three things are to be proved: injectivity, surjectivity and compatibility with the monoid structures of the Bézout map. Each one has an elementary proof. In $\$ 3.4 .1$, we start by proving the surjectivity and the compatibility condition, both at the same time. We then remark in $\$ 3.4 .2$ that injectivity reduces to that of Béz $z_{2}$. We conclude in $\$ 3.4 .3$ by an independent analysis of the scheme $\mathscr{F}_{2}$ and of the map Béz $z_{2}$. 
3.4.1. Surjectivity and compatibility with sums. - We adopt the following conventions.

Definition 3.12. - Let $n$ be a positive integer. For a sequence of scalars $u_{1}, \ldots, u_{n}$ in $k^{\times}$, let

$-\left\langle u_{1}, \ldots, u_{n}\right\rangle$ denote the diagonal symmetric bilinear form $\left\langle u_{1}\right\rangle \oplus \cdots \oplus\left\langle u_{n}\right\rangle \in \varnothing_{n}(k)$.

- $\left[u_{1}, \ldots, u_{n}\right]$ denote the pointed rational function $\frac{X}{u_{1}} \oplus^{\mathrm{N}} \cdots \oplus^{\mathrm{N}} \frac{X}{u_{n}} \in \mathcal{F}_{n}(k)$.

The following lemma shows that, up to naive homotopy, any symmetric bilinear form and any rational function is of the preceding form.

Lemma 3.13. - Let $n$ be a positive integer. Then:

1. For any symmetric bilinear form $S \in \phi_{n}(k)$ there exist units $u_{1}, \ldots, u_{n} \in k^{\times}$such that $S$ is homotopic to the diagonal form $\left\langle u_{1}, \ldots, u_{n}\right\rangle$.

2. For any pointed rational function $f \in \mathcal{F}_{n}(k)$ there exist units $u_{1}, \ldots, u_{n} \in k^{\times}$such that we have

$$
f \stackrel{p}{\sim}\left[u_{1}, \ldots, u_{n}\right]
$$

Proof. - $\quad$ 1. Suppose first that $\operatorname{char}(k) \neq 2$. Then every symmetric matrix $S \in \phi_{n}(k)$ is congruent by an element $P \in \mathbf{S L}_{n}(k)$ to a diagonal matrix. Decomposing $P$ into a product of elementary matrices yields a homotopy, i.e., an element of $\phi_{n}(k[T])$, to a diagonal matrix.

When $\operatorname{char}(k)=2$, then $S$ is congruent by an element $P \in \mathbf{S L}_{n}(k)$ to a block diagonal matrix, with possible $\left[\begin{array}{ll}0 & 1 \\ 1 & 0\end{array}\right]$ terms. One can use homotopies of the form $\left[\begin{array}{ll}T & 1 \\ 1 & 0\end{array}\right]$ to link $S$ to a diagonalizable matrix and then repeat the preceding argument.

2. We prove this point by induction on the degree $n$ of $f$.

As noted in Example 3.3, a rational function $f \in \mathcal{F}_{n}(k)$ is tautologically the $\oplus^{\mathrm{N}}$-sum of some polynomials, say $f=P_{1} \oplus^{\mathrm{N}} \cdots \oplus^{\mathrm{N}} P_{k}$. It is thus enough to treat the case when $f$ is a polynomial. We can even assume that $f$ is a monomial $\frac{X^{n}}{u}$ (with $u \in k^{\times}$) as any polynomial is homotopic to its leading term. The element $\frac{X^{n}}{T X^{n-1}+u} \in \mathcal{F}_{n}(k[T])$ defines a homotopy between $\frac{X^{n}}{u}$ and $\frac{X^{n}}{X^{n-1}+u}$. But this last rational function decomposes as $X \oplus^{\mathrm{N}} g$ for some $g \in \mathcal{F}_{n-1}(k)$. One concludes by using the inductive hypothesis on $g$.

The monoids $\left(\left(\pi_{0}^{\mathrm{N}} \ell\right)(k), \oplus\right)$ and $\left(\left(\pi_{0}^{\mathrm{N}} \mathcal{F}\right)(k), \oplus^{\mathrm{N}}\right)$ are generated by their degree $1 \mathrm{com}$ ponents. Since the map $\pi_{0}^{\mathrm{N}} \mathrm{Béz}_{1}:\left(\pi_{0}^{\mathrm{N}} \mathcal{F}_{1}\right)(k) \longrightarrow\left(\pi_{0}^{\mathrm{N}} \phi_{1}\right)(k)$ is a bijection, the following lemma shows that the Bézout form of a rational function of the form $\left[u_{1}, \ldots, u_{n}\right] \in \mathcal{F}_{n}(k)$ is homotopic to the diagonal form $\left\langle u_{1}, \ldots, u_{n}\right\rangle$.

Lemma 3.14. - Let $\frac{A}{B} \in \mathcal{F}_{n}(k)$ and $u \in k^{\times}$. Then the Bézout form of $\frac{X}{u} \oplus^{\mathrm{N}} \frac{A}{B}$ is congruent by an element in $\mathbf{S L}_{n+1}(k)$ (and is thus also homotopic) to the block diagonal form $\langle u\rangle \oplus \operatorname{Béz}_{n}(A, B)$. 
Proof. - By definition, one has $\frac{X}{u} \oplus^{\mathrm{N}} \frac{A}{B}=\frac{X A-\frac{B}{u}}{u A}$. Using the notations introduced in Definition 3.4, we have

$$
\delta_{X A-\frac{B}{u}, u A}(X, Y)=u A(X) A(Y)+\delta_{A, B}(X, Y) .
$$

In the basis $\left(1, X, \ldots, X^{n-1}, A(X)\right)$, the matrix of the Bézout form is diagonal as announced.

This proves that the Bézout map induces a surjective morphism of monoids.

3.4.2. Injectivity. - Let $n$ be a positive integer. To prove the injectivity of the map $\pi_{0}^{\mathrm{N}}$ Béz $_{n}$, we prove the injectivity of the composite

$$
\left(\pi_{0}^{\mathrm{N}} \mathcal{F}_{n}\right)(k) \stackrel{\pi_{0}^{\mathrm{N}} \mathrm{Béz}_{n}}{\longrightarrow}\left(\pi_{0}^{\mathrm{N}} \phi_{n}\right)(k) \stackrel{\bar{q}_{n} \times \operatorname{det}}{\longrightarrow} \mathrm{MW}_{n}^{\mathrm{s}}(k) \underset{k^{\times} / k^{\times 2}}{\times} k^{\times} .
$$

Because up to homotopy any rational function is a $\oplus^{\mathrm{N}}$-sum of degree 1 monomials $(c f$. Lemma 3.13), the injectivity of the previous map can be reformulated as follows.

Proposition 3.15. - Let $u_{1}, \ldots, u_{n}, v_{1}, \ldots, v_{n}$ be a list of units in $k^{\times}$. If the classes in $\mathrm{MW}_{n}^{\mathrm{s}}(k) \underset{k^{\times} / k^{\times 2}}{\times} k^{\times}$of the diagonal forms $\left\langle u_{1}, \ldots, u_{n}\right\rangle$ and $\left\langle v_{1}, \ldots, v_{n}\right\rangle$ are equal, then $\left[u_{1}, \ldots, u_{n}\right] \stackrel{\mathrm{p}}{\sim}\left[v_{1}, \ldots, v_{n}\right]$ holds in $\mathcal{F}_{n}(k)$.

Proof. - We introduce some ad hoc terminology. Two diagonal forms $\left\langle u_{1}, \ldots, u_{n}\right\rangle$ and $\left\langle v_{1}, \ldots, v_{n}\right\rangle$ are said to be equivalent through an elementary $\mathbf{S L}_{2}(k)$-transformation if there exists an integer $1 \leqslant i \leqslant n-1$ such that:

- the 2-forms $\left\langle u_{i}, u_{i+1}\right\rangle$ and $\left\langle v_{i}, v_{i+1}\right\rangle$ are $\mathbf{S L}_{2}(k)$-equivalent;

- for all $j \neq i, i+1$, we have $u_{j}=v_{j}$.

The next lemma is a slight reformulation of [8, Lemma III.5.6], which gives a presentation of the Witt group $\mathrm{W}(k)$ by generators and relations.

Lemma 3.16. - Let $\left\langle u_{1}, \ldots, u_{n}\right\rangle$ and $\left\langle v_{1}, \ldots, v_{n}\right\rangle$ be two diagonal forms in $\phi_{n}(k)$. Their images in $\mathrm{MW}_{n}^{\mathrm{s}}(k) \underset{k^{\times} / k^{\times 2}}{\times} k^{\times}$are equal if and only if one can "pass from one to the other" by a finite sequence of elementary $\mathbf{S L}_{2}(k)$-transformations.

Therefore, in order to prove Proposition 3.15, it is enough to deal with the case $n=2$. This case is analyzed independently in the next paragraph.

4 e SÉRIE - TOME $45-2012-$ No $^{\circ}$ 
3.4.3. Rational functions of degree 2. - Let $\mathbf{G}_{\mathbf{a}}$ be the "additive group", that is to say the affine line $\mathbf{A}^{1}$ seen as a group scheme. For every positive integer $n, \mathbf{G}_{\mathbf{a}}$ acts freely on $\mathcal{F}_{n}$ by translations, i.e., by the formula

$$
h \cdot \frac{A}{B}:=\frac{A+h B}{B},
$$

on the level of points. The following lemma shows that the $\mathbf{G}_{\mathbf{a}}$-torsor $\mathcal{F}_{n}$ splits.

Lemma 3.17. - Let $R$ be a ring and $\frac{A}{B}$ be an element of $\mathcal{F}_{n}(R)$. There exists a unique pair of polynomials $\left(U_{1}, V_{1}\right)$ of $R[X]$ with $\operatorname{deg}\left(U_{1}\right)=n-1, \operatorname{deg}\left(V_{1}\right) \leqslant n-1$ and such that $A U_{1}+B V_{1}=X^{2 n-1}$. Let $\phi_{n}\left(\frac{A}{B}\right)$ be the opposite of the coefficient of $X^{n-1}$ in $V_{1}$. Then the associated scheme morphism $\phi_{n}: \mathcal{F}_{n} \longrightarrow \mathbf{A}^{1}$ is $\mathbf{G}_{\mathbf{a}}$-equivariant. In particular, $\mathcal{F}_{n}$ splits as the product $\phi_{n}^{-1}(0) \times \mathbf{A}^{1}$.

Proof. - Let $A, B, U_{1}$ and $V_{1}$ be as above. If one changes $(A, B)$ to $(A+h B, B)$, then $\left(U_{1}, V_{1}\right)$ is changed to $\left(U_{1}, V_{1}-h U_{1}\right)$. The claim follows since $U_{1}$ is monic.

Moreover, observe that the morphism Béz $z_{n}: \mathscr{F}_{n} \longrightarrow \mathcal{\ell}_{n}$ is by construction $\mathbf{G}_{\mathbf{a}}$-equivariant when $\phi_{n}$ is endowed with the trivial action. In dimension 2 , the morphism Béz 2 induces an isomorphism between $\phi_{2}^{-1}(0)$ and $\phi_{2}$.

Proposition 3.18. - The morphism

$$
\mathcal{F}_{2} \stackrel{\text { Béź }_{2} \times \phi_{2}}{\longrightarrow} \oint_{2} \times \mathbf{A}^{1}
$$

is a $\mathbf{G}_{\mathbf{a}}$-equivariant isomorphism of schemes.

COROLlary 3.19. - The following map is injective:

$$
\left(\pi_{0}^{\mathrm{N}} \mathcal{F}_{2}\right)(k) \stackrel{\pi_{0}^{\mathrm{N}} \mathrm{Béz}_{2}}{\longrightarrow}\left(\pi_{0}^{\mathrm{N}} \oint_{2}\right)(k) \stackrel{\bar{q}_{2} \times \operatorname{det}}{\longrightarrow} \mathrm{MW}_{2}^{\mathrm{s}}(k) \underset{k^{\times} / k^{\times 2}}{\times} k^{\times} .
$$

Corollary 3.19 concludes the proof of Proposition 3.15.

Proof of Proposition 3.18. - One can write down the inverse morphism $\psi: \phi_{2} \longrightarrow \phi_{2}^{-1}(0)$ by solving a system of two equations with two unknowns. The formula is:

$$
\psi\left(\left[\begin{array}{ll}
\alpha & \beta \\
\beta & \gamma
\end{array}\right]\right)=\frac{X^{2}+\frac{\alpha \beta}{\beta^{2}-\alpha \gamma} X+\frac{\alpha^{2}}{\beta^{2}-\alpha \gamma}}{\gamma X+\beta} .
$$

The proof of Theorem 3.6 is now complete. 


\subsection{Comparing naive and motivic homotopy classes}

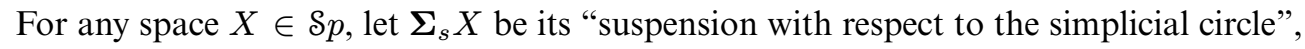
that is to say

$$
\boldsymbol{\Sigma}_{s} X:=\operatorname{hocolim}\left({ }^{\mathrm{pt}} \longrightarrow_{X} \nearrow^{\mathrm{pt}}\right)
$$

Lemma 3.20. - There is a canonical homotopy equivalence:

$$
\mathbf{P}^{1} \approx \boldsymbol{\Sigma}_{s}\left(\mathbf{A}^{1}-\{0\}\right) .
$$

Proof. - This is a consequence of $\mathbf{P}^{1}$ being covered by the two contractible open subschemes $\mathbf{P}^{1}-\{0\}$ and $\mathbf{P}^{1}-\{\infty\}$, intersecting along $\mathbf{P}^{1}-\{\infty, 0\} \simeq \mathbf{A}^{1}-\{0\}$.

The preceding lemma endows the set $\left[\mathbf{P}^{1}, \mathbf{P}^{1}\right]^{\mathbf{A}^{1}}$ with a group structure, whose law is denoted by $\oplus^{\mathbf{A}^{1}}$. The canonical map $\left[\mathbf{P}^{1}, \mathbf{P}^{1}\right]^{\mathrm{N}} \longrightarrow\left[\mathbf{P}^{1}, \mathbf{P}^{1}\right]^{\mathbf{A}^{1}}$ is thus not a bijection: it can reach only elements of non-negative degrees. However, Morel's result shows that the error in the naive computation is as small as possible.

THEOREM 3.21 (Morel, [11], Theorem 6.36). - There is a group isomorphism

$$
\left(\left[\mathbf{P}^{1}, \mathbf{P}^{1}\right]^{\mathbf{A}^{1}}, \oplus^{\mathbf{A}^{1}}\right) \simeq\left(\mathrm{GW}(k) \underset{k^{\times} / k^{\times 2}}{\times} k^{\times}, \oplus\right) .
$$

In particular, this implies that the group $\left(\left[\mathbf{P}^{1}, \mathbf{P}^{1}\right]^{\mathbf{A}^{1}}, \oplus^{\mathbf{A}^{1}}\right)$ is abstractly isomorphic to the group completion of the monoid $\left(\left[\mathbf{P}^{1}, \mathbf{P}^{1}\right]^{\mathrm{N}}, \oplus^{\mathrm{N}}\right)$. We show next that, as one can expect, the group completion is induced by the canonical map from the naive to the $\mathbf{A}^{1}$-homotopy classes.

Theorem 3.22. - The canonical map $\left(\left[\mathbf{P}^{1}, \mathbf{P}^{1}\right]^{\mathrm{N}}, \oplus^{\mathrm{N}}\right) \rightarrow\left(\left[\mathbf{P}^{1}, \mathbf{P}^{1}\right]^{\mathbf{A}^{1}}, \oplus^{\mathbf{A}^{1}}\right)$ is a group completion.

Proof. - The main point is to prove the compatibility between the monoid law $\oplus^{\mathrm{N}}$ on $\left[\mathbf{P}^{1}, \mathbf{P}^{1}\right]^{\mathrm{N}}$ and the group law $\oplus^{\mathbf{A}^{1}}$ on $\left[\mathbf{P}^{1}, \mathbf{P}^{1}\right]^{\mathbf{A}^{1}}$. Namely, we have the following:

Proposition 3.23. - The canonical map $\left(\left[\mathbf{P}^{1}, \mathbf{P}^{1}\right]^{\mathrm{N}}, \oplus^{\mathrm{N}}\right) \longrightarrow\left(\left[\mathbf{P}^{1}, \mathbf{P}^{1}\right]^{\mathbf{A}^{1}}, \oplus^{\mathbf{A}^{1}}\right)$ is a monoid morphism.

A detailed proof of Proposition 3.23 is postponed until the appendix because it would be too digressive here.

By universal property, the canonical map $\left[\mathbf{P}^{1}, \mathbf{P}^{1}\right]^{\mathrm{N}} \rightarrow\left[\mathbf{P}^{1}, \mathbf{P}^{1}\right]^{\mathbf{A}^{1}}$ factors through the group completion of $\left[\mathbf{P}^{1}, \mathbf{P}^{1}\right]^{\mathrm{N}}$, say $\operatorname{Gr}\left(\left[\mathbf{P}^{1}, \mathbf{P}^{1}\right]^{\mathrm{N}}\right)$. Since Morel's Theorem 3.21 indicates that $\operatorname{Gr}\left(\left[\mathbf{P}^{1}, \mathbf{P}^{1}\right]^{\mathrm{N}}\right)$ and $\left[\mathbf{P}^{1}, \mathbf{P}^{1}\right]^{\mathbf{A}^{1}}$ are abstractly isomorphic groups, we have to check that the degree 1 generators in $\operatorname{Gr}\left(\left[\mathbf{P}^{1}, \mathbf{P}^{1}\right]^{\mathrm{N}}\right)$ are sent to the degree 1 generators in $\left[\mathbf{P}^{1}, \mathbf{P}^{1}\right]^{\mathbf{A}^{1}}$. 
Let $a \in k^{\times}$be a unit, let $f$ be the rational function $\frac{X}{a}$ and let $\pi(f)$ denote the image of $f$ in $\left[\mathbf{P}^{1}, \mathbf{P}^{1}\right]^{\mathbf{A}^{1}}$. Morel's isomorphism (3.21) is described in [11, p. 225-226]. With the following identifications:

$$
\begin{aligned}
\mathrm{GW}(k) & \simeq K_{0}^{\mathrm{MW}}(k) \simeq \operatorname{End}\left(\underline{\mathbf{K}}_{1}^{\mathrm{MW}}\right) \\
\text { and } \mathrm{H}_{1}^{\mathbf{A}^{1}}\left(\mathbf{P}^{1}\right) & \simeq \mathrm{H}_{1}^{\mathbf{A}^{1}}\left(\boldsymbol{\Sigma}_{s} \mathbf{G}_{\mathbf{m}}\right) \simeq \tilde{\mathrm{H}}_{0}^{\mathbf{A}^{1}}\left(\mathbf{G}_{\mathbf{m}}\right) \simeq \underline{\mathbf{K}}_{1}^{\mathrm{MW}}
\end{aligned}
$$

the component of $\pi(f)$ in $\mathrm{GW}(k)$ is given by its "degree" $\mathrm{H}_{1}^{\mathbf{A}^{1}}(f) \in \operatorname{End}\left(\underline{\mathbf{K}}_{1}^{\mathrm{MW}}\right)$. But the morphism $f$ is homotopy equivalent to the suspension $\boldsymbol{\Sigma}_{s} \tilde{f}$ of the morphism $\tilde{f}: \mathbf{G}_{\mathbf{m}} \stackrel{\times \frac{1}{a}}{\longrightarrow} \mathbf{G}_{\mathbf{m}}$, whose degree $\tilde{\mathrm{H}}_{0}^{\mathbf{A}^{1}}(\tilde{f})$ is checked(5) to be the generator $\left\langle\frac{1}{a}\right\rangle$ in $\mathrm{GW}(k)$.

\section{Related computations}

The previous computation has natural extensions which we consider now.

- In $\$ 4.1$, we compute the set of free homotopy classes of endomorphisms of $\mathbf{P}^{1}$.

- In $\S 4.2$, we make explicit the monoid structure induced on $\left[\mathbf{P}^{1}, \mathbf{P}^{1}\right]^{\mathrm{N}}$ by the composition of endomorphisms of $\mathbf{P}^{1}$.

- Finally, in $\$ 4.3$, we compute the set of naive homotopy classes of pointed morphisms from $\mathbf{P}^{1}$ to $\mathbf{P}^{d}$ for every $d \geqslant 2$.

\subsection{Free homotopy classes of rational functions}

We compute here the set $\left\{\mathbf{P}^{1}, \mathbf{P}^{1}\right\}^{\mathrm{N}}$ of naive homotopy classes of free (i.e., unpointed) endomorphisms of $\mathbf{P}^{1}$. The result is mostly a consequence of the previous computation of $\left[\mathbf{P}^{1}, \mathbf{P}^{1}\right]^{\mathrm{N}}$.

The description of free endomorphisms of $\mathbf{P}^{1}$ in terms of rational functions is the following.

Definition 4.1. - For every non-negative integer $n$, the scheme $\mathscr{U}_{n}$ of unpointed degree $n$ rational functions is the open subscheme of $\mathbf{P}^{2 n+1}:=\operatorname{Proj}\left(k\left[a_{0}, \ldots, a_{n}, b_{0}, \ldots, b_{n}\right]\right)$ complementary to the hypersurface of equation

$$
\operatorname{res}_{n, n}\left(a_{n} X^{n}+\cdots+a_{0}, b_{n} X^{n}+\cdots+b_{0}\right)=0 .
$$

Proposition 4.2. - Let $R=k$ or $R=k[T]$. The datum of a $k$-scheme morphism $f: \mathbf{P}_{R}^{1} \longrightarrow \mathbf{P}^{1}$ is equivalent to the datum of a non-negative integer $n$ (its degree) and of an element in $\mathscr{U}_{n}(R)$.

We denote by $\stackrel{\mathrm{u}}{\sim}$ the equivalence relation generated by unpointed naive homotopies and by $\left\{\mathbf{P}^{1}, \mathbf{P}^{1}\right\}^{\mathrm{N}}$ the set of unpointed naive homotopy classes of endomorphisms of $\mathbf{P}^{1}$. As before, the degree is a naive homotopy invariant and the set $\left\{\mathbf{P}^{1}, \mathbf{P}^{1}\right\}^{\mathrm{N}}$ splits degree wise:

$$
\left\{\mathbf{P}^{1}, \mathbf{P}^{1}\right\}^{\mathrm{N}}=\coprod_{n \geqslant 0}\left\{\mathbf{P}^{1}, \mathbf{P}^{1}\right\}_{n}^{\mathrm{N}}
$$

(5) Be careful that the morphism $\tilde{f}$ is unpointed. 
Let $n$ be a positive integer. Note that a $k$-point in $\mathscr{U}_{n}$ gives a pair of coprime polynomials $(A, B)$ only up to the multiplication by a unit of $k$. Thus the Bézout form of an unpointed endomorphism of $\mathbf{P}^{1}$ is defined only up to multiplication by the square of a unit of $k$. We are going to prove that the class of the Bézout form is an invariant that distinguishes exactly the homotopy classes:

THEOREM 4.3. - The canonical map of graded sets:

$$
\begin{aligned}
\left\{\mathbf{P}^{1}, \mathbf{P}^{1}\right\}^{\mathrm{N}}=\coprod_{n \geqslant 0}\left\{\mathbf{P}^{1}, \mathbf{P}^{1}\right\}_{n}^{\mathrm{N}} & \longrightarrow \coprod_{n \geqslant 0} \operatorname{MW}_{n}^{\mathrm{s}}(k){ }_{k^{\times} / k^{\times 2}} \times k^{\times} / k^{\times 2 n} \\
{\left[\frac{A}{B}\right] } & \mapsto[\operatorname{Béz}(A, B), \operatorname{det} \operatorname{Béz}(A, B)]
\end{aligned}
$$

is a bijection.

Proof of Theorem 4.3. - This is a consequence of Corollary 3.10 and of the following lemma.

Lemma 4.4. - $\quad$ 1. Any free rational function is naively homotopic to a pointed rational function.

2. Let $f$ and $g$ be two pointed rational functions. Then one has the relation $f \stackrel{u}{\sim} g$ if and only if there exists a non-zero element $\lambda \in k^{\times}$with $f \stackrel{\mathrm{p}}{\sim} \lambda^{2} g$.

Proof. - $\quad$ 1. Let $f=\frac{A}{B}$ represent a rational function and let $\alpha_{1}$ be a matrix in $\mathbf{S L}_{2}(k)$ such that $\alpha_{1} \cdot \infty=f(\infty)$, for the usual action of $\mathbf{S L}_{2}(k)$ on $\mathbf{P}^{1}(k)$. Let $\alpha(T)$ be an algebraic path in $\mathbf{S L}_{2}(k[T])$ linking the identity to $\alpha_{1}$. (Again, this can be obtained using a decomposition of $\alpha_{1}$ as a product of elementary matrices). The column vector $\alpha(T)^{-1} \cdot\left(\begin{array}{l}A \\ B\end{array}\right)$ is a $k[T]$-point of $\mathscr{U}_{n}$ and thus yields a homotopy between $f$ and the pointed rational function $\alpha_{1}^{-1} \cdot \frac{A}{B}$.

2. Suppose first that we have two rational functions $f, g$ such that $f \stackrel{\mathrm{u}}{\sim} g$ and let us show that there exists a unit $\lambda \in k^{\times}$such that $f \stackrel{\mathrm{p}}{\sim} \lambda^{2} g$.

Let

$$
f=f_{0} \underset{F_{0}(T)}{\stackrel{u}{\sim}} f_{1} \underset{F_{1}(T)}{\stackrel{u}{\sim}} \cdots \underset{F_{N-1}(T)}{\stackrel{u}{\sim}} f_{N}=g
$$

be a sequence of elementary homotopies between $f$ and $g$. Let $\alpha(T)$ be a matrix in $\mathbf{S L}_{2}(k[T])$ such that we have $F_{0}(T, \infty)=\alpha(T) \cdot \infty$ and $\alpha(0)=$ id. The path $\alpha(T)^{-1} \cdot F_{0}(T)$ yields a pointed homotopy between the pointed rational functions $f_{0}$ and $\alpha(1)^{-1} \cdot f_{1}$. Moreover, for $N>1$, we then have a sequence of $N-1$ free homotopies

$$
\alpha(1)^{-1} \cdot f_{1} \underset{\alpha(1-T)^{-1} F_{1}(T)}{\sim} f_{2} \underset{F_{2}(T)}{\sim} \cdots \underset{F_{N-1}(T)}{\sim} f_{N}=g .
$$

Thus the result will follow by induction from the case $N=1$.

When $N=1, \alpha(1)^{-1}$ is of the form $\left[\begin{array}{cc}\lambda & \mu \\ 0 & \lambda^{-1}\end{array}\right]$. So, $\left[\begin{array}{cc}1 & T \lambda \mu \\ 0 & 1\end{array}\right] \cdot \alpha(T)^{-1} \cdot F_{0}(T)$ gives a pointed homotopy between $f_{0}$ and $\lambda^{2} f_{1}$. 
We now show the converse. For every $\lambda \in k^{\times}$, a path in $\mathbf{S L}_{2}(k[T])$ between the identity matrix and $\left[\begin{array}{cc}\lambda & 0 \\ 0 & \lambda^{-1}\end{array}\right]$ yields a free homotopy between any rational function $g$ and $\lambda^{2} g$. The result follows.

\subsection{Composition of rational functions}

The set $\left[\mathbf{P}^{1}, \mathbf{P}^{1}\right]^{\mathrm{N}}$ admits a second monoid structure induced by composition of morphisms. We make explicit this new structure in terms of our previous description of $\left[\mathbf{P}^{1}, \mathbf{P}^{1}\right]^{\mathrm{N}}$.

Definition 4.5. - Define a new composition law, say o, on $\underset{n \geqslant 0}{\coprod} \mathrm{MW}_{n}^{\mathrm{s}}(k) \underset{k^{\times} / k^{\times 2}}{\times} k^{\times}$by

$$
\left(b_{1}, \lambda_{1}\right) \circ\left(b_{2}, \lambda_{2}\right):=\left(b_{1} \otimes b_{2}, \lambda_{1}^{\operatorname{dim} b_{2}} \lambda_{2}^{\left(\operatorname{dim} b_{1}\right)^{2}}\right) .
$$

This law is associative and endows $\coprod_{n \geqslant 0} \mathrm{MW}_{n}^{\mathrm{s}}(k) \underset{k^{\times} / k^{\times 2}}{\times} k^{\times}$with a monoid structure.

Theorem 4.6. - The following map induces an isomorphism of graded "bi-monoids"

$$
\begin{aligned}
\left(\left[\mathbf{P}^{1}, \mathbf{P}^{1}\right]^{\mathrm{N}}, \oplus^{\mathrm{N}}, \circ\right) & \longrightarrow\left(\coprod_{n \geqslant 0} \operatorname{MW}_{n}^{\mathrm{s}}(k) \underset{k^{\times} / k^{\times 2}}{\times} k^{\times}, \oplus, \circ\right) \\
f & \mapsto[\operatorname{Béz}(f), \operatorname{res}(f)]
\end{aligned}
$$

Remark 4.7. - Warning: the triple $\left(\left[\mathbf{P}^{1}, \mathbf{P}^{1}\right]^{\mathrm{N}}, \oplus^{\mathrm{N}}, \mathrm{o}\right)$ is not a semi-ring. In general, one has distributivity of o over $\oplus^{\mathrm{N}}$ only on the left-hand side: in general for a triple $\left(f, g_{1}, g_{2}\right)$ of pointed rational functions, $\left(g_{1} \oplus^{\mathrm{N}} g_{2}\right) \circ f$ and $\left(g_{1} \circ f\right) \oplus^{\mathrm{N}}\left(g_{2} \circ f\right)$ are not equal in $\left[\mathbf{P}^{1}, \mathbf{P}^{1}\right]^{\mathrm{N}}$.

Proof of Theorem 4.6. - Since any pointed rational function is up to homotopy a $\oplus^{\mathrm{N}}$-sum of degree 1 rational functions, Theorem 4.6 is a consequence of the following lemma.

Lemma 4.8. - Let $a \in k^{\times}$be a unit, $m$ and $n$ be positive integers, and $f=\frac{A}{B} \in \mathcal{F}_{m}(k)$ and $g=\frac{C}{D} \in \mathcal{F}_{n}(k)$ be two pointed rational functions. Then

1. We have $\frac{X}{a} \circ f=\frac{1}{a} f$.

2. In the stable Witt monoid $\mathrm{MW}_{(m+1) n}^{\mathrm{s}}(k)$, we have:

$$
\operatorname{Béz}_{(m+1) n}\left(\left(X \oplus^{\mathrm{N}} f\right) \circ g\right)=\operatorname{Béz}_{n}(g) \oplus^{\mathrm{N}} \operatorname{Béz}_{m n}(f \circ g) .
$$

3. $\operatorname{det} \mathrm{Béz}_{(n+1) m}\left(\left(X \oplus^{\mathrm{N}} f\right) \circ g\right)=\operatorname{det} \mathrm{Béz}_{n}(g)^{2 m+1} \cdot \operatorname{det} \mathrm{Béz}_{n m}(f \circ g)$.

Proof. - 1. This is true by definition. 
2. Let $\frac{\tilde{A}}{\tilde{B}}$ be the pointed rational function representing $f \circ g$. By definition, one has:

$$
\tilde{A}(X)=\sum_{i=0}^{m} a_{i} C(X)^{i} D(X)^{m-i} \quad \text { and } \quad \tilde{B}(X)=\sum_{i=1}^{m} b_{i} C(X)^{i} D(X)^{m-i} .
$$

Since we have $X \oplus^{\mathrm{N}} \frac{A}{B}=X-\frac{B}{A}$, the endomorphism $\left(X \oplus^{\mathrm{N}} f\right) \circ g$ is represented by the rational function

$$
\frac{C}{D}-\frac{\tilde{B}}{\tilde{A}}=\frac{C \tilde{A}-D \tilde{B}}{D \tilde{A}} .
$$

Moreover, using the notations of Definition 3.4, we have the identity

$$
\delta_{C \tilde{A}-D \tilde{B}, D \tilde{A}}(X, Y)=\tilde{A}(X) \tilde{A}(Y) \delta_{C, D}(X, Y)+D(X) D(Y) \delta_{\tilde{A}, \tilde{B}}(X, Y) .
$$

Observing the congruence $\tilde{A} \equiv C^{m} \bmod D$, we deduce

$$
\operatorname{res}(\tilde{A}, D)=\operatorname{res}\left(C^{m}, D\right)=\operatorname{res}(C, D)^{m} \in k^{\times} .
$$

Thus $\left(D(X), X \cdot D(X), \ldots, X^{m n-1} \cdot D(X), \tilde{A}(X), X \cdot \tilde{A}(X), \ldots, X^{m-1} \tilde{A}(X)\right)$ gives a basis of the $k$-vector space of polynomials of degree $<(n+1) m-1$. And in this basis, the matrix of the form Béz $(n+1) m\left(\left(X \oplus^{\mathrm{N}} f\right) \circ g\right)$ is

$$
\left[\begin{array}{cc}
\operatorname{Béz}_{m n}(\tilde{A}, \tilde{B}) & 0 \\
0 & \operatorname{Béz}_{m}(C, D)
\end{array}\right] .
$$

3. This point follows from the proof of the previous one. Indeed, we have just proved the following matrix identity

$$
\operatorname{Béz}_{(n+1) m}(C \tilde{A}-D \tilde{B}, D \tilde{A})={ }^{t} \operatorname{Syl}(\tilde{A}, D)\left[\begin{array}{cc}
\operatorname{Béz}_{m n}(\tilde{A}, \tilde{B}) & 0 \\
0 & \operatorname{Bézz}_{m}(C, D)
\end{array}\right] \operatorname{Syl}(\tilde{A}, D) \text {, }
$$

where Syl is the Sylvester matrix (see $\left.\left[1, \S 6, \mathrm{n}^{\circ} 6, \mathrm{IV}\right]\right)$. The result now follows from the relation $\operatorname{det} \operatorname{Syl}(\tilde{A}, D)=\operatorname{res}(\tilde{A}, D)=\operatorname{res}(C, D)^{m}$.

This completes the proof of Theorem 4.6.

\subsection{Naive homotopy classes of morphisms to higher dimensional projective spaces}

Let $d \geqslant 2$ be an integer. We compute now the naive homotopy classes of $k$-scheme morphisms from $\mathbf{P}^{1}$ to $\mathbf{P}^{d}$. The group of $\mathbf{A}^{1}$-homotopy classes of morphisms from $\mathbf{P}^{1}$ to $\mathbf{P}^{d}$ is also determined in [11] and we can again compare our result to it. Not surprisingly, the computation is much easier than the previous one for $d=1$.

For brevity, we treat only the case of pointed morphisms. The base point in $\mathbf{P}^{d}$ is taken at $\infty:=[1: 0: \ldots: 0]$. Pointed scheme morphisms from $\mathbf{P}^{1}$ to $\mathbf{P}^{d}$ and naive homotopies between them admit the following concrete description, analogous to that of Proposition 2.3.

Definition 4.9. - For every non-negative integer $n$, let $\mathcal{F}_{n}^{d}(-)$ be the functor from the category of $k$-algebras to the category of sets which maps any $k$-algebra $R$ to the set of pairs $(A, B)$, where $A$ is a monic polynomial of $R[X]$ of degree $n$, and $B:=\left(B_{1}, \ldots, B_{d}\right)$ is a $d$-tuple of polynomials, each of degree strictly less than $n$ and such that the ideal generated by the family $\left\{A, B_{1}, \ldots, B_{d}\right\}$ is $R[X]$. These functors $\mathcal{F}_{n}^{d}(-)$ are representable by smooth schemes, which we denote by $\mathcal{F}_{n}^{d}$ again. 
Proposition 4.10. - Let $R=k$ or $R=k[T]$. The datum of a pointed $k$-scheme morphism $f: \mathbf{P}_{R}^{1} \longrightarrow \mathbf{P}^{d}$ is equivalent to the datum of a non-negative integer $n$ (its degree) and of an element in $\mathcal{F}_{n}^{d}(R)$.

Remark 4.11. - Let $R$ be a ring and $A$ be a monic polynomial in $R[X]$. The datum of a $B$ such that $(A, B)$ is an $R$-point of $\mathcal{F}_{n}^{d}$ is equivalent to the datum of an element

$$
\left(\overline{B_{1}}, \ldots, \overline{B_{d}}\right) \in\left(\mathbf{A}^{d}-\{0\}\right)(R[X] /(A)) .
$$

This point of view leads to the definition of Atiyah-Hitchin schemes, see [4, 3].

As in Definition 2.5 , we denote by $\stackrel{p}{\sim}$ the equivalence relation generated by pointed naive homotopies of morphisms from $\mathbf{P}^{1}$ to $\mathbf{P}^{d}$ and by $\left[\mathbf{P}^{1}, \mathbf{P}^{d}\right]^{\mathrm{N}}$ the corresponding set of naive homotopy classes. The degree of morphisms is again invariant through naive homotopies; we are going to prove that this is the only invariant.

Theorem 4.12. - For every $d \geqslant 2$, the degree map $\operatorname{deg}:\left[\mathbf{P}^{1}, \mathbf{P}^{d}\right]^{\mathrm{N}} \stackrel{\simeq}{\longrightarrow} \mathbf{N}$ is a bijection.

Proof. - Fix a non-negative integer $n$. We are going to prove that the set $\left(\pi_{0}^{\mathrm{N}} \mathcal{F}_{n}^{d}\right)(k)$ contains only one element. More precisely, we are going to link any element $\left(A, B_{1}, \ldots, B_{d}\right) \in$ $\mathcal{F}_{n}^{d}(k)$ to $\left(X^{n}, 1, \ldots, 1\right)$ by a sequence of pointed naive homotopies.

Note first that it is enough to $\operatorname{link}\left(A, B_{1}, \ldots, B_{d}\right)$ to $(A, 1, \ldots, 1)$ since the pointed homotopy $\left((1-T) A+T X^{n}, 1, \ldots, 1\right) \in \mathcal{F}_{n}^{d}(k[T])$ will then link $(A, 1, \ldots, 1)$ to $\left(X^{n}, 1, \ldots, 1\right)$.

To do so, decompose the polynomial $A$ as a product of irreducible factors:

$$
A=\prod_{i=1}^{r} P_{i}^{r_{i}} .
$$

As noticed in Remark 4.11, the set of $k$-points of $\mathcal{F}_{n}^{d}$ with first coordinate equal to $A$ is in bijection with $\left(\mathbf{A}^{d}-\{0\}\right)(k[X] /(A))$. The Chinese remainder theorem identifies this set with

$$
\prod_{i=1}^{r}\left(\mathbf{A}^{d}-\{0\}\right)\left(k[X] /\left(P_{i}^{r_{i}}\right)\right) .
$$

It is therefore enough to treat the case when $A$ is a power of an irreducible polynomial, say $A=P^{r}$. In this case, the $\operatorname{ring} R:=k[X] /\left(P^{r}\right)$ is local. A $d$-tuple $\left(\overline{B_{1}}, \ldots, \overline{B_{d}}\right) \in R^{n}$ represents an element of $\left(\mathbf{A}^{d}-\{0\}\right)\left(k[X] /\left(P^{r}\right)\right)$ if and only if one of the $\overline{B_{i}}$ is a unit of $R$. Up to reordering, we can assume that $\overline{B_{1}}$ is such a unit. The element $\left(A, B_{1},(1-T) B_{2}+T, \ldots,(1-T) B_{d}+T\right) \in \mathcal{F}_{n}^{d}(k[T])$ then yields a pointed homotopy linking $\left(A, B_{1}, B_{2}, \ldots, B_{d}\right)$ to $\left(A, B_{1}, 1, \ldots, 1\right)$. One concludes using the pointed homotopy $\left(A,(1-T) B_{1}+T, 1, \ldots, 1\right)$ from $\left(A, B_{1}, 1, \ldots, 1\right)$ to $(A, 1,1, \ldots, 1)$.

As in $\S 3.5,\left[\mathbf{P}^{1}, \mathbf{P}^{d}\right]^{\mathbf{A}^{1}}$ has a natural group law denoted by $\oplus^{\mathbf{A}^{1}}$. Morel's result is the following.

Theorem 4.13 (Morel, [11], Theorem 6.13). - For every integer $d \geqslant 2$, the degree map

$$
\left(\left[\mathbf{P}^{1}, \mathbf{P}^{d}\right]^{\mathbf{A}^{1}}, \oplus^{\mathbf{A}^{1}}\right) \stackrel{\operatorname{deg}}{\longrightarrow}(\mathbf{Z},+)
$$

induces a group isomorphism. 
It follows from Theorem 4.12 that the set $\left[\mathbf{P}^{1}, \mathbf{P}^{d}\right]^{\mathrm{N}}$ is a fortiori endowed with a monoid structure, pulled back from that of $(\mathbf{N},+)$. We denote again its law by $\oplus^{\mathrm{N}}$. Naive and $\mathbf{A}^{1}$-homotopy classes compare again very well.

COROllary 4.14. - For every integer $d \geqslant 2$, the canonical map

$$
\left(\left[\mathbf{P}^{1}, \mathbf{P}^{d}\right]^{\mathrm{N}}, \oplus^{\mathrm{N}}\right) \longrightarrow\left(\left[\mathbf{P}^{1}, \mathbf{P}^{d}\right]^{\mathbf{A}^{1}}, \oplus^{\mathbf{A}^{1}}\right)
$$

is a group completion.

\section{Appendix}

\section{Additions of rational functions}

The goal of this appendix is to compare the two addition laws on homotopy classes of endomorphisms of $\mathbf{P}^{1}$ : the naive law denoted by $\oplus^{\mathrm{N}}$ defined in $\S 3.1$ and the $\mathbf{A}^{1}$ law, coming from $\mathbf{P}^{1}$ being a suspension. More precisely, we are going to prove Proposition 3.23, that is:

Proposition A.1. - The canonical map

$$
\left(\left[\mathbf{P}^{1}, \mathbf{P}^{1}\right]^{\mathrm{N}}, \oplus^{\mathrm{N}}\right) \longrightarrow\left(\left[\mathbf{P}^{1}, \mathbf{P}^{1}\right]^{\mathbf{A}^{1}}, \oplus^{\mathbf{A}^{1}}\right)
$$

is a monoid morphism.

Whereas the $\oplus$ law is very concrete, the $\oplus^{\mathbf{A}^{1}}$ law is somehow more delicate to make explicit, since writing the coproduct $\nabla: \mathbf{P}^{1} \rightarrow \mathbf{P}^{1} \vee \mathbf{P}^{1}$ involves inverting several weak homotopy equivalences in $\mathcal{S} p$. Therefore, our first step is to give a workable model for this coproduct.

Definition A.2. - Let $\mathbf{P}^{1} \coprod_{0 \sim \infty} \mathbf{P}^{1}$ be the cofiber of the map $S^{0}=\{0\} \amalg\{\infty\} \hookrightarrow \mathbf{P}^{1} \amalg \mathbf{P}^{1}$. (Equivalently, $\mathbf{P}^{1} \coprod_{0 \sim \infty} \mathbf{P}^{1}$ is the union of two copies of $\mathbf{P}^{1}$ with the point 0 in the first copy identified with the point $\infty$ in the second one). The base point is taken at $\infty$ in the first copy.

The following map $\tilde{\nabla}: \mathbf{P}^{1} \rightarrow \mathbf{P}^{1} \coprod_{0 \sim \infty} \mathbf{P}^{1}$ is a "model" for the unreduced coproduct of $\mathbf{P}^{1}$ :

$$
\tilde{\nabla}: \mathbf{P}^{1} \longrightarrow \mathbf{P}^{1} / \mathbf{P}^{1}-\{0, \infty\}=\mathbf{P}^{1} / \mathbf{P}^{1}-\{\infty\}_{* \sim *}^{\amalg} \mathbf{P}^{1} / \mathbf{P}^{1}-\{0\} \stackrel{\approx}{\approx} \mathbf{P}^{1} \coprod_{0 \sim \infty} \mathbf{P}^{1}
$$

corresponding to the following picture:
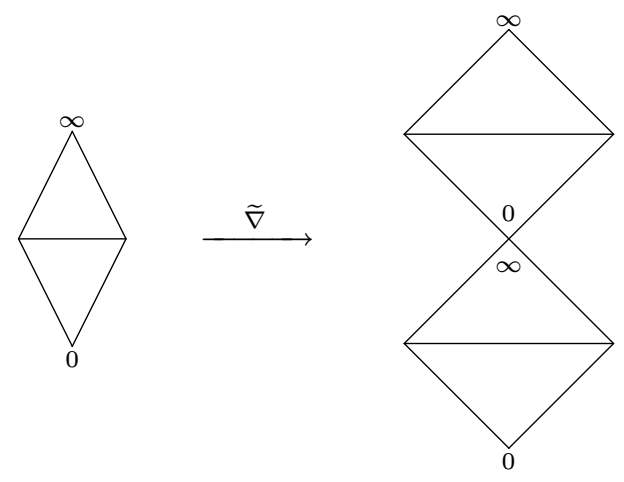

$4^{\text {e }}$ SÉRIE - TOME $45-2012-\mathrm{N}^{\circ} 4$ 
The definition of $\tilde{\nabla}$ requires an explanation. The first and the third maps in (A.2) are the natural projection maps, whereas $*$ is a generic notation for the canonical point in a quotient. The (abusive) equal sign "=" in the middle of (A.2) refers to a canonical isomorphism of spaces (not just a homotopy equivalence). Indeed, we will use the following fact several times.

Lemma A.3 (Compare with Lemma 2.1.13 in [10]). - Let $X$ be a smooth scheme and $Z_{1}$, $Z_{2}$ be two disjoint closed subschemes. Then there is a canonical isomorphism of spaces in $\mathrm{Sp}$ :

$$
X / X-\left(Z_{1} \coprod Z_{2}\right)=X / X-Z_{1} \vee X / X-Z_{2}
$$

Finally, note that the map $\tilde{\nabla}$ is compatible with the reduced coproduct $\nabla$, in the sense where the following diagram of pointed spaces homotopy commutes:

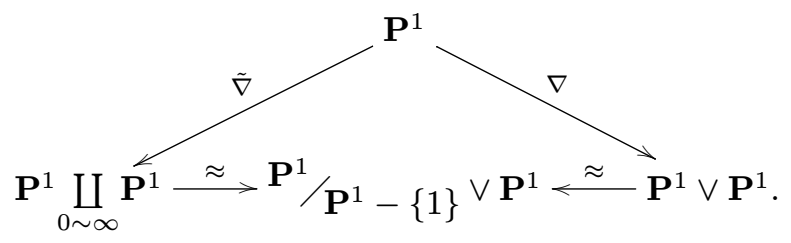

Proof of Proposition A.1. - Let $g_{1}$ and $g_{2}$ be two pointed rational functions. We need to prove that the image of the rational function $g_{1} \oplus^{\mathrm{N}} g_{2}$ in $\left[\mathbf{P}^{1}, \mathbf{P}^{1}\right]^{\mathbf{A}^{1}}$ is equal to $g_{1} \oplus^{\mathbf{A}^{1}} g_{2}$. Since the monoid $\left[\mathbf{P}^{1}, \mathbf{P}^{1}\right]^{\mathrm{N}}$ of naive homotopy classes of rational functions is generated by its elements of degree 1 ( $c f$. Lemma 3.13), it is enough to deal with the case when $g_{1}$ is of degree 1 . Up to a canonical homotopy, one can even assume that $g_{1}$ is of the form $\frac{X}{a}$ for some $a \in k^{\times}$. For $g_{1}$ of this form, the formula for the $\oplus^{\mathrm{N}}$-sum is

$$
\frac{X}{a} \oplus^{\mathrm{N}} g_{2}=\frac{X}{a}-\frac{1}{a^{2}} \frac{1}{g_{2}} \text {. }
$$

Lemma A.4. - Let $g: \mathbf{P}^{1} \longrightarrow \mathbf{P}^{1}$ be a pointed rational function and let $f$ be the pointed rational function $f=\frac{X}{a}-\frac{1}{g}$. One has $f^{-1}(\infty)=\{\infty\} \amalg g^{-1}(0)$ and we denote by $\bar{f}$ the induced map between the cofibers:

$$
\bar{f}: \mathbf{P}^{1} / \mathbf{P}^{1}-\left(\{\infty\} \coprod g^{-1}(0)\right) \longrightarrow \mathbf{P}^{1} / \mathbf{P}^{1}-\{\infty\} .
$$

Then the following diagram of pointed spaces in $\mathrm{S} p$ homotopy commutes:

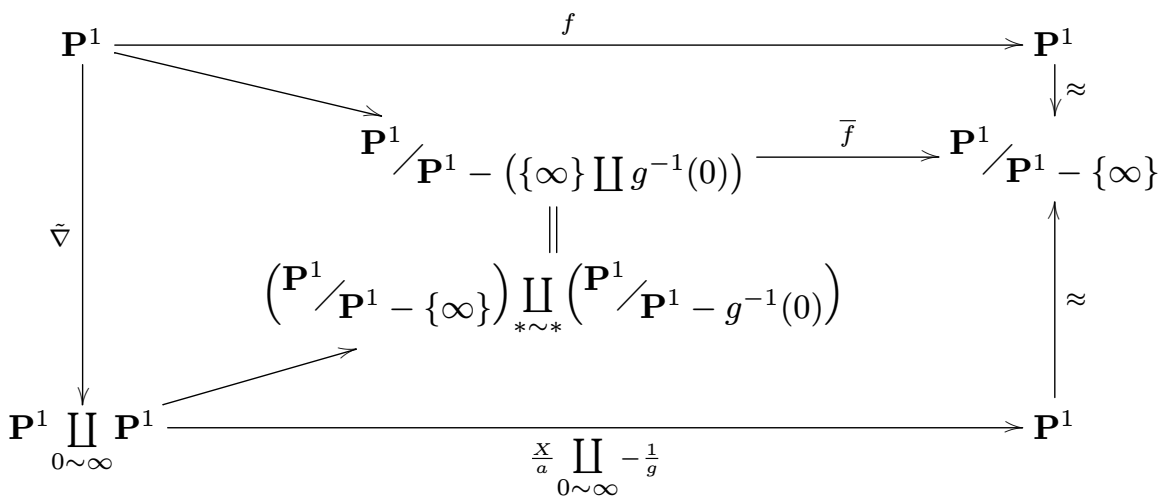


(The two diagonal arrows are projection maps and the equal sign refers to Lemma A.3.)

Proof. - To check commutativity of the trapezoid on the left-hand side one has to "separate" $\infty$ and $g^{-1}(0)$ inside $\mathbf{P}^{1}$. This can be done by inverting the homotopy equivalence of pointed spaces $\mathbf{P}^{1} \underset{\approx}{\approx} \mathbf{A}^{1} \coprod_{0 \sim \infty} \mathbf{P}^{1}$ (the base point in this latter space is $1 \in \mathbf{A}^{1}$ ).

The main point is then to prove that the following two diagrams:

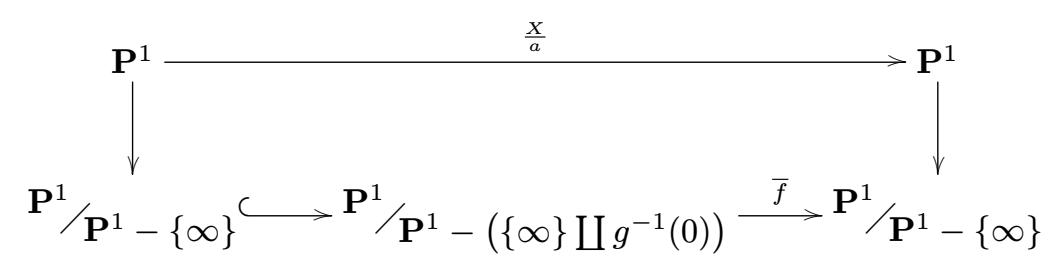

and

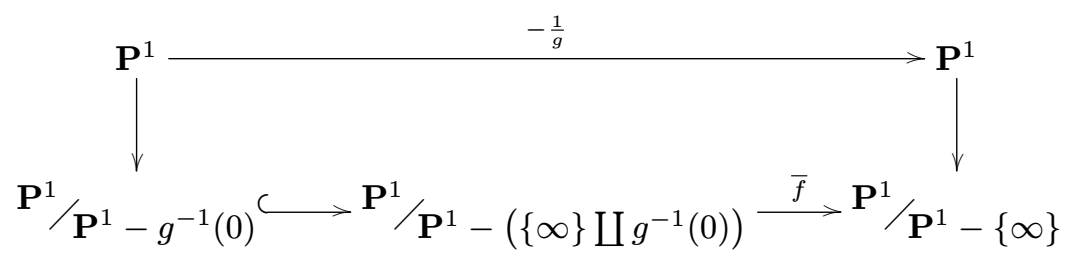

homotopy commute. The proof is similar in both cases, so we detail only the second one.

Since $g^{-1}(0) \cap\{\infty\}=\varnothing$, the canonical map of pairs $\left(\mathbf{A}^{1}, \mathbf{A}^{1}-g^{-1}(0)\right) \hookrightarrow\left(\mathbf{P}^{1}, \mathbf{P}^{1}-g^{-1}(0)\right)$ induces an isomorphism $\mathbf{A}^{1} / \mathbf{A}^{1}-g^{-1}(0)=\mathbf{P}^{1} / \mathbf{P}^{1}-g^{-1}(0)$ (cf. Lemma A.3). But there is a naive homotopy of morphisms of pairs:

$$
\begin{aligned}
\left(\mathbf{A}^{1}, \mathbf{A}^{1}-g^{-1}(0)\right) \times \mathbf{A}^{1} & \longrightarrow\left(\mathbf{P}^{1}, \mathbf{P}^{1}-\{\infty\}\right) \\
(X, T) & \mapsto T \frac{X}{a}-\frac{1}{g(X)}
\end{aligned}
$$

between $f_{\mid\left(\mathbf{A}^{1}, \mathbf{A}^{1}-g^{-1}(0)\right)}$ and $\left(-\frac{1}{g}\right)_{\mid\left(\mathbf{A}^{1}, \mathbf{A}^{1}-g^{-1}(0)\right)}$, which implies the homotopy commutativity of the diagram.

Lemma A.4 reduces the proof of Proposition A.1 to expressing the composite map

$$
\mathbf{P}^{1} \stackrel{\tilde{\nabla}}{\longrightarrow} \mathbf{P}^{1} \coprod_{0 \sim \infty} \mathbf{P}^{1} \stackrel{\frac{x}{a} \coprod_{0 \sim \infty}-\frac{1}{g}}{\longrightarrow} \mathbf{P}^{1}
$$

as a sum in the group $\left[\mathbf{P}^{1}, \mathbf{P}^{1}\right]^{\mathbf{A}^{1}}$. The result is given in the next lemma, which is stated in the following context:

- Let $Y$ be the unreduced suspension of a pointed space $\left(\tilde{Y}, y_{0}\right)$, that is to say

$$
Y:=\operatorname{hocolim}(\mathrm{pt} \longleftarrow \tilde{Y} \longrightarrow \mathrm{pt}) \text {. }
$$

The space $Y$ has two distinguished points, denoted by $\infty$ and 0 , linked by the canonical path hocolim (pt $\longleftarrow\left\{y_{0}\right\} \longrightarrow \mathrm{pt}$ ). The base point in $Y$ is taken at $\infty$.

- Let $\left(Z ; z_{0}\right)$ be an $\mathbf{A}^{1}$-connected pointed space.

- Let $g_{1}, g_{2}: Y \longrightarrow Z$ be two maps such that $g_{1}(0)=g_{2}(\infty)$. We assume that $g_{1}$ is pointed, that is to say that $g_{1}(\infty)=z_{0}$. 
- Let $f$ be the pointed map $\left(g_{1} \underset{0 \sim \infty}{\amalg} g_{2}\right) \circ \tilde{\nabla}: Y \longrightarrow Z$.

- Let $H: Y \times \mathbf{A}^{1} \longrightarrow Z$ be a naive homotopy from $g_{2}$ to a pointed map, say $g_{3}: Y \longrightarrow Z$.

- Let $\alpha: \mathbf{A}^{1} \longrightarrow Z$ be the path $T \mapsto H(\infty, T)$ in $Z$ from $g_{2}(\infty)$ to $z_{0}$.

- Let $\beta: \mathbf{A}^{1} \longrightarrow Z$ be the image through $g_{1}$ of the canonical path in $Y$ from $\infty$ to 0 ; $\beta$ is thus a path in $Z$ from $z_{0}$ to $g_{1}(0)=g_{2}(\infty)$.

- Let $\gamma$ be the concatenation of the paths $\beta$ and $\alpha ; \gamma$ is thus a loop in $Z$ at $z_{0}$.

Lemma A.5. - In the group $[Y, Z]^{\mathbf{A}^{1}}$, one has the identity:

$$
\gamma \cdot f=\left(\gamma \cdot g_{1}\right) \oplus^{\mathbf{A}^{1}} g_{3} .
$$

Above here, the dots "." denote the action of an element of $\pi_{1}^{\mathbf{A}^{1}}\left(Z ; z_{0}\right)$ on $[Y, Z]^{\mathbf{A}^{1}}$.

Proof. - It is a consequence of the following facts:

- Up to homotopy, $Y$ can be replaced by $\mathbf{A}^{1} \coprod_{0 \sim \infty} Y$, pointed at $1 \in \mathbf{A}^{1}$. The element $\gamma \cdot f$ is represented by the map
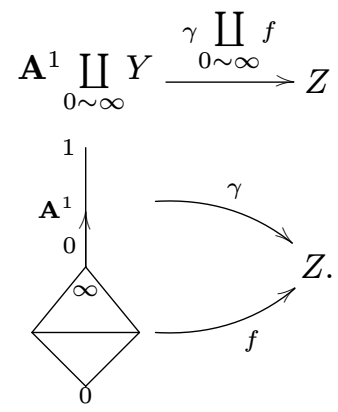

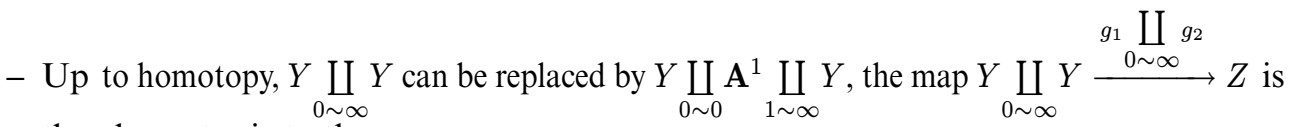
then homotopic to the map

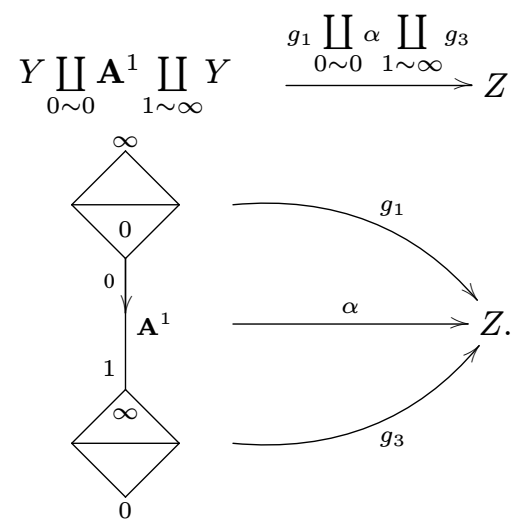

- The restriction of the map

$$
\mathbf{A}^{1} \coprod_{0 \sim \infty} Y \coprod_{0 \sim 0} \mathbf{A}^{1} \underset{1 \sim \infty}{\amalg} Y \stackrel{\gamma \underset{0 \sim \infty}{\amalg} f \coprod_{0 \sim 0} \alpha \underset{1 \sim \infty}{\amalg} g_{3}}{\longrightarrow} Z
$$


to $\mathbf{A}^{1} \coprod_{0 \sim 0} \mathbf{A}^{1} \coprod_{1 \sim 0} \mathbf{A}^{1}$ (the $\mathbf{A}^{1}$ in the middle is the domain of $\beta$ ) is the concatenation of $\gamma^{-1}$ and $\gamma$ and is thus null-homotopic. So, up to homotopy, the map $\gamma \underset{0 \sim \infty}{\amalg} f \coprod_{0 \sim 0} \alpha \underset{1 \sim \infty}{\amalg} g_{3}$ factors through the cofiber $\left(\mathbf{A}^{1} \underset{0 \sim \infty}{\amalg} Y \coprod_{0 \sim 0} \mathbf{A}^{1} \underset{1 \sim \infty}{\amalg} Y\right) /\left(\mathbf{A}^{1} \coprod_{0 \sim 0} \mathbf{A}^{1} \coprod_{1 \sim 0} \mathbf{A}^{1}\right) \approx Y \vee Y$.

- The following composite map

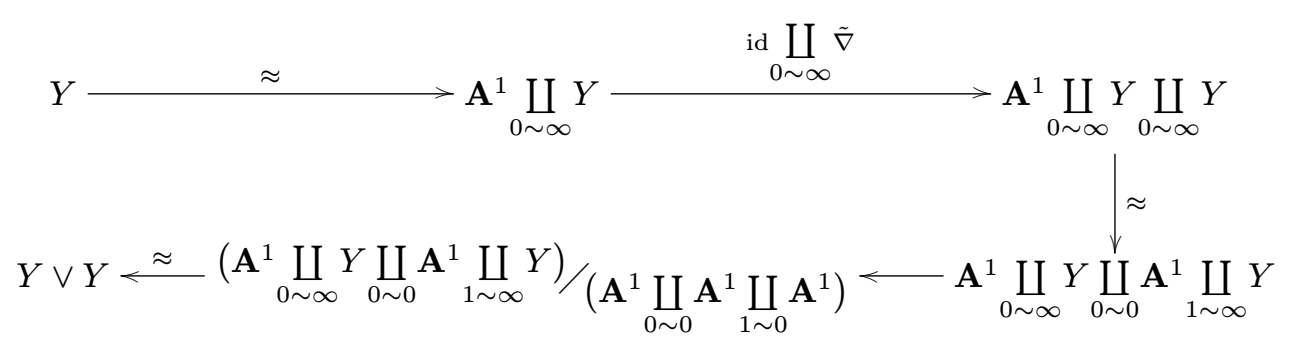

is homotopic to the coproduct $Y \stackrel{\nabla}{\longrightarrow} Y \vee Y$.

In our situation, there is a "universal" homotopy between $-\frac{1}{g}$ and $g$, which is given by composition at the target of $g$ with a naive homotopy between between $-\frac{1}{X}$ and $X$. One can choose

$$
\frac{\left(-T^{2}+2 T\right) X-\left(T^{3}-3 T^{2}+T+1\right)}{(-T+1) X+\left(-T^{2}+2 T\right)}
$$

as an example of such a homotopy.

Lemma A.6. - Let $\alpha: \mathbf{A}^{1} \longrightarrow \mathbf{P}^{1}$ be the path $T \mapsto\left[-T^{2}+2 T:-T+1\right], \beta: \mathbf{A}^{1} \longrightarrow \mathbf{P}^{1}$ be the path $T \mapsto[1-T: a T]$ and $\gamma$ be the loop given by concatenation of $\alpha$ and $\beta$. Then for any pointed rational function $f: \mathbf{P}^{1} \longrightarrow \mathbf{P}^{1}$, one has a canonical homotopy between $\gamma \cdot f$ and $a^{2} f$.

Proof. - We can lift the paths $\alpha$ and $\beta$ in $\mathbf{S L}_{2}$ and use the product in $\mathbf{S L}_{2}$ to compose them. More precisely, let $\tilde{\beta}: \mathbf{A}^{1} \longrightarrow \mathbf{S L}_{2}$ be a lift of $\beta$ such that $\tilde{\beta}(0)=$ id and let $\tilde{\alpha}: \mathbf{A}^{1} \longrightarrow \mathbf{S L}_{2}$ be a lift of $\alpha$ such that $\tilde{\alpha}(0)=\tilde{\beta}(1)$. Then a lift $\tilde{\gamma}$ of the loop $\gamma$ is

$$
\begin{aligned}
\tilde{\gamma}: \mathbf{A}^{1} & \longrightarrow \mathbf{S L}_{2} \\
T & \mapsto \tilde{\beta}(T) \cdot \tilde{\beta}(1)^{-1} \cdot \tilde{\alpha}(T) .
\end{aligned}
$$

Let $c_{\infty}: \mathbf{A}^{1} \longrightarrow \mathbf{P}^{1}$ be the constant path at $\infty \in \mathbf{P}^{1}$. The action of $\mathbf{S L}_{2}$ on $\mathbf{P}^{1}$ can be used to define a naive homotopy between $\gamma \underset{0 \sim \infty}{\amalg} f$ and $c_{\infty} \underset{0 \sim \infty}{\amalg}(\tilde{\alpha}(1) \cdot f)$, namely ${ }^{(6)}$

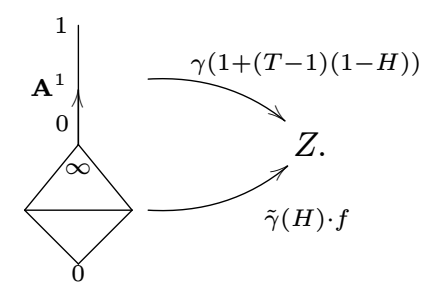

(6) Below, $H \in \mathbf{A}^{1}$ is the parameter of the homotopy and $T \in \mathbf{A}^{1}$ belongs to the domain of $\gamma$. 
Explicitly, one can take $\tilde{\alpha}(T)=\left[\begin{array}{cc}a\left(-T^{2}+2 T\right) & \left(-2+\frac{1}{a}\right) T^{2}+\left(4-\frac{1}{a}\right) T-\frac{1}{a} \\ a(-T+1) & \left(-2+\frac{1}{a}\right) T+2\end{array}\right]$ and $\tilde{\beta}(T)=\left[\begin{array}{cc}1-T & -\frac{T}{a} \\ a T & 1+T\end{array}\right]$. So $\tilde{\gamma}(1)=\tilde{\alpha}(1)=\left[\begin{array}{cc}a & 2-\frac{1}{a} \\ 0 & \frac{1}{a}\end{array}\right]$. The result follows since $\tilde{\gamma}(1) \cdot f=$ $a^{2} f+2-\frac{1}{a}$ is canonically homotopic to $a^{2} f$.

Lemma A.5 and Lemma A.6 together imply Proposition A.1. Indeed, for every $a \in k^{\times}$ and for every pointed rational function $g$ one has the identity in $\left[\mathbf{P}^{1}, \mathbf{P}^{1}\right]^{\mathbf{A}^{1}}$

$$
a X \oplus^{\mathrm{N}} g=a X-\frac{a^{2}}{g}=a^{2}\left(\frac{X}{a}-\frac{1}{g}\right)=a X \oplus^{\mathbf{A}^{1}} g .
$$

Remark A.7. - Our proof of Proposition A.1 produces a homotopy between $\frac{X}{a} \oplus^{\mathrm{N}} g$ and $\frac{X}{a} \oplus \mathbf{A}^{1} g$ which is uniform in $a \in k^{\times}$and $g \in \mathcal{F}_{n}$. Therefore for every positive integer $n$ the following diagram of spaces homotopy commutes:

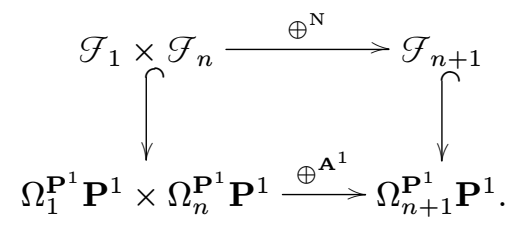

Above, $\Omega^{\mathbf{P}^{1}} \mathbf{P}^{1}$ is the (derived) space of pointed endomorphisms of $\mathbf{P}^{1}$. This space splits degree wise and we denote by $\Omega_{k}^{\mathbf{P}^{1}} \mathbf{P}^{1}$ its degree $k$ component (which naturally contains $\mathcal{F}_{k}$ as a subspace).

\section{BIBLIOGRAPHY}

[1] N. Bourbaki, Éléments de mathématique. Algèbre, chapitre IV: Polynômes et fractions rationnelles, Hermann, Paris, 1950 ; réédition Springer, 2007.

[2] C. Cazanave, Classes d'homotopie de fractions rationnelles, C. R. Math. Acad. Sci. Paris 346 (2008), 129-133.

[3] C. Cazanave, Théorie homotopique des schémas d'Atiyah-Hitchin, thèse de doctorat, Université Paris 13, 2009.

[4] C. Cazanave, The $\mathbf{A}^{1}$-homotopy type of Atiyah-Hitchin schemes I: The geometry of complex points, preprint, 2010.

[5] I. M. Gel'fand, M. M. Kapranov, A. V. Zelevinsky, Discriminants, resultants, and multidimensional determinants, Mathematics: Theory \& Applications, Birkhäuser, 1994.

[6] M. Karoubi, O. Villamayor, $K$-théorie algébrique et $K$-théorie topologique. I, Math. Scand. 28 (1971), 265-307.

[7] T. Y. Lam, Serre's problem on projective modules, Springer Monographs in Math., Springer, 2006.

[8] J. Milnor, D. Husemoller, Symmetric bilinear forms, Ergebn. Math. Grenzg. 73, Springer, 1973. 
[9] F. Morel, Théorie homotopique des schémas, Astérisque 256 (1999).

[10] F. Morel, An introduction to $\mathbb{A}^{1}$-homotopy theory, in Contemporary developments in algebraic K-theory, ICTP Lect. Notes, XV, Abdus Salam Int. Cent. Theoret. Phys., Trieste, 2004, 357-441 (electronic).

[11] F. Morel, $\mathbf{A}^{1}$-algebraic topology over a field, Lecture Notes in Math. 2052, Springer, 2012.

[12] F. Morel, V. Voevodsky, $\mathbf{A}^{1}$-homotopy theory of schemes, Publ. Math. I.H.É.S. 90 (1999), 45-143.

[13] D. G. Quillen, Homotopical algebra, Lecture Notes in Math., No. 43, Springer, 1967.

Manuscrit reçu le 25 décembre 2009 ;

accepté, après modification, le 20 avril 2012.)

\author{
Christophe Cazanave \\ Univérsité de Nice Sophia-Antipolis \\ Laboratoire J.-A. Dieudonné \\ CNRS UMR 7351 \\ Parc Valrose \\ 06108 Nice Cedex 02, France \\ E-mail: cazanave@unice.fr
}

